\title{
The Research Progress and Suggestion Strategy of Health Service Accessibility
}

\author{
Meilin Xiang \\ College of Health and Conservation, Tianfu College of Southwestern University of Finance and Economics, \\ Chengdu,610052, China \\ Corresponding Author's Email: 577969326@qq.com
}

\begin{abstract}
Health service accessibility plays an important role in formulation of health policy. Recently, though related research on health service accessibility is increasing at home and abroad, there are still many deficiencies of them. The paper mainly summarizes conception, definition, research status and existing problems of health service accessibility, and puts forward suggestion strategy in view of the current drawbacks, thus providing an empirical basis for recommendation of theory development and improvement of policy practice.
\end{abstract}

Keywords: Health Service Accessibility, Research Progress, Suggestion Strategy.

\section{INTRODUCTION}

As a significant index in the health service system, health service accessibility is utilized to balance and evaluate its fairness, efficiency and quality condition. [1] The earliest conception of "accessibility" origins from the related research on health field, come up with by a scholar named Anderson in 1968 and described as "the progress of continued use after entering into health service system by any means." [2] Most of the later references all indicate health service accessibility is relevant to timely acquirement of corresponding health service in the light of people's demand. [3] WHO gives the definition of health service accessibility: difficulty level for residents to meet their basic medical and health demand in the space. [4] Currently, in China, the government defines basic public health service accessibility as the demand of the public for basic public health service and match situation of government's supplies for them as well as use condition of the public for basic public health service. [5]

\section{THE ROLE AND SIGNIFICANCE OF HEALTH SERVICE ACCESSIBILITY}

As a bridge, the health service accessibility can connect with three elements of health results, health service and people to understand the analysis of fairness, quality and efficiency of health service. [6] However, comparing with the attention to the research on health service accessibility and related aspects exception of personal level at abroad in recent years, the research at home is limited and lack of depth and foresight. According to the policy documents issued by the Central Committee, improvement of basic public health service accessibility, as the breakthrough and focus of governments of all levels to promote the supply side structural reform of basic public services, is the new topics for related researchers to be urgently issued. Strengthen the research on health service accessibility can provide real and important reference basis for the nation to adjust health policy, and come up with significant reference opinions for improvement of people's health service quality. Therefore, the paper summarizes current research progress at home and abroad and sums up the research status of basic public health service to give corresponding suggestions and strategies for existing problems.

\section{RESEARCH STATUS OF BASIC PUBLIC HEALTH SERVICE ACCESSIBILITY AT ABROAD}

Research on basic public health service accessibility at abroad mainly includes three aspects of evaluation index, meaning and impact factors of health service accessibility.[7] As for the research on evaluation index, Anderson initially divided the health service accessibility into six categories in accordance with his theory model. [2] Aday L A \& Andersen R put forward five characteristic indexes for health service accessibility evaluation in 1974 and later Peters D H and others 
classified the health service accessibility into four dimensions. [8-9] For the research on obstacle factors affecting the utilization of health services, Derose and others added the genetic inheritance factor and environment factor after concluding previous model basis and put forward latest accessibility research framework [10] in 2011 that is still in constant improvement and development at present. With regard to the research on the conception of health service accessibility, there are different opinions among scholars at abroad. From focusing on health service visit capacity to fairness and personal free choice, of which the conception also becomes rich and extended. [11-13] Many scholars at abroad hold that the influence factors of health service accessibility are various, which are mainly showed on the respects of individuality, economy, organization and policy as well as social environment support, such as residents' health condition on individuality, income level and medical fee on economy condition, supply condition of medical care on organization, policy and social environment support as well as policy characteristics, all impacting on the health service accessibility development. [14-15]

\section{RESEARCH STATUS OF BASIC PUBLIC HEALTH SERVICE ACCESSIBILITY AT HOME}

On the basis of learning and summing up Western health service accessibility theory, the scholars at home unfolds the research on the different types of basic public service accessibility problems, mainly focusing on researching on influencing factors and countermeasures, and obtaining rich research results. With accordance to related data of health service in Hubei Province, Gao Xiang and others [16] analyzes the reason of differences between urban and rural areas in the aspects of basic medical services accessibility for the elderly in Hubei Province, and gives corresponding suggestions about how to narrow the income gap of the elderly between urban and rural areas, adjust the allocation of medical resource and expand medical service supply. Combined with realistic condition, Huang Dujuan and others [17] construct 3D lifting model of basic public health service accessibility and gives corresponding solutions for the purpose of improving accessibility. On the basis of medical service utilization behavior model, Du Benfeng and others [18] mainly analyzes and discusses the utilization conditions of health and health services of mobile population in Beijing in 2015 from three aspects of demand, supply and utilization of medical health service, and gives corresponding health promoting strategies in the view of supply side and utilization. Jiang Yingying and others [19] utilizes dynamic monitoring data of mobile population in Shanghai in 2016 to analyze influence factors of basic medical health service accessibility of mobile population in Shanghai in the four respects of geographic accessibility, availability, economic affordability and acceptability. Though existing research has achieved a lot of valued results, from which we can find there are obvious imbalance and insufficiency among the study, for example, domestic basic public service accessibility research still has limitation of research content, conception not comforting with national conditions and measurement tools lack of theorization and other problems in some degree. Therefore, based on the existing research, weak links need to be strengthened and deepen.

\section{THE DEFICIENCY AND PROBLEMS OF THE HEALTH SERVICE ACCESSIBILITY IN CHINA}

\subsection{Definition lack of a unified standard}

At present, the experts and scholars at home and abroad still do not give a unified standard to significant terms in the health field about the research on health service accessibility, which is not favorable to deeper study on health service accessibility. Though most references at present show health service accessibility is relevant to timely acquired health care service.[20] However, there are few studies on its standard and unified definition and conception development and definition lack of localization.[21-22] Due to current research do not unfold systemic comparison and sorting out the theories between China and other nations, present health service accessibility theory research in China is relatively poor.

\subsection{Imperfect research method}

Quantitative research is mostly applied to health service accessibility, but at present the scholars at home and abroad still do not establish a set of object and easy evaluation index systems. In China, evaluation index systems of health service for community mainly use the structure-process-result model, and index selection is mainly Delphi method, along with deficiency in the aspects of integration, reliability and validity test of subjectivity and objectivity. On the discussion of influence factors of research to service accessibility, most researches adopt simple data analysis method without logical theoretical analysis framework, thus it is impossible to conduct deep analysis and discussion on research.

\subsection{Research status lack of depth}

Currently, domestic study reference of health service accessibility is much fewer and the study range is also relatively limited. At abroad, more references generally focus on other factors related with health service accessibility besides personal aspects and impacting on health service accessibility, such as gene, health service quality and nationality, etc. [23] By comparison, health 
service accessibility research in China is more limited, which is mostly from overall level of some areas or certain group of people to analyze the influence factors of health service accessibility by common test data. One is lack of in-deep research on regions choice; another is restricted selection of people groups. At present, it is more attention to the elderly people and mobile population but less discussion and focus on other special population, thus it is hard to reflect nationwide condition, generally, lacking of depth. [24]

\subsection{More implementation barriers of health service accessibility, poor implementation effect, policy needs to be improved}

Currently, the implementation of health service accessibility in China includes the following aspects: (1) the difference resulted by regions of health service accessibility is larger. The present study shows the difference of health service accessibility in central and western regions in China is much larger. Compared with the urban, the rural has a smaller organization barrier, but economic barriers seriously block the spreading and development of rural health service accessibility, including conflict between lower growth rates of household per capita income and faster rising of medical service price as well as lower medical insurance ownership rate. [25] (2) The government gives less attention and imbalanced investment to the health development. Firstly, on capital input, recent data show the government's health expenditure accounted for $27.5 \%$, just $7.5 \%$ higher than the data recommended by WHO, but individual spending on health $10 \%$ higher than the government, which is heavier burden. Meanwhile, rural areas accounted for $32.8 \%$, while urban areas accounted for $67.2 \%$ in the total composition of health expenditure. There are serious differences in the distribution of expenditure between rural and urban areas. Most of the national capital investment is oriented to urban areas, while the rural primary health service investment is relatively lower. [26](3) Inadequate medical facilities. The rural remote area is far from the medical institution with poor facilities. The fifth National Health Service investigation shows [27] the condition of long distance from poor rural areas to hospital with poor medical equipment has been improved, but the gap between urban and rural areas still exists. (4) The talent allocation of health service accessibility needs to be improved, and the quantity of medical workers engaged in health services is smaller and the medical team is unstable. While investigating the rural medical personnel in Fujian Province, Chen Shaofeng[28] found that the medical team in rural areas is composed of people who are mainly engaged in agriculture and other industries, which is very unstable. Even there are villages in the remote areas with poor economic development and inconvenient transportation without rural doctors. (5) Implementation policy of health service accessibility needs to be improved. Firstly, the propaganda conducing of health service accessibility has not played its role in China, so that related knowledge of health service accessibility is unable to be accepted by people, which is not favorable to gain recognition from people. Secondly there are defects in the medical security and assistance system, thus leading to the rural residents cannot afford higher medical fee and impeding to the development of health service accessibility.[29]

\section{CONCLUSION\&STRATEGY TO IMPROVE AND ENHANCE THE HEALTH SERVICE ACCESSIBILITY IN CHINA}

\subsection{Conception operationalization of basic public health service accessibility}

In order to promote the substantial progress of research on health service accessibility, we should suggest unified standard and utilize operationalization to convert conception into available index, in the process of which the relation between internationalization and localization as well as individuality and generality should be balanced. Though operationalization of health service accessibility in the west has been a more mature framework after decades' development, accessibility in China has its special practical direction, which should be comfort with the socialist system with Chinese characteristics. Therefore, based on national actual condition, domestic researchers should develop conception operation method with localization by using excellent results at abroad for reference.

At present, the conception of health service accessibility mainly gained from related research on health service field, though involving in other fields, but conception framework of health service accessibility is also basically followed to use. Therefore, we should adjust the relations between individuality and generality of health service accessibility and other basic public health services as well as considering difference and particularity among them.

\subsection{Research on the causal mechanism of promoting the basic public services accessibility}

Measuring basic public health service accessibility is the basis of empirical research. On the one hand, we need to perfect research method and research theory system, such as depth research its cause and effect, thus it is favorable to promote theoretical study and know what factors to effect basic public service accessibility and its influence so as to effectively promote policy and practice improvement. 


\subsection{Extending key fields and groups of research on basic public health service accessibility}

Firstly, the research on key fields should be enlarged. Though research results of health service accessibility in China is not less, the research on accessibility problems in the key public service fields should be conducted when the research is continuously promoted, such as the fields of public education and elderly care, etc,. In the process of fighting against COVID-19, many problems of national medical and health service accessibility are exposed, from which the theory circle can conduct its research on accessibility.

Secondly, the research on key groups should be strengthened. On policy making, the state should regard grass-roots masses as key people, whether it aims at promoting basic public health service equalization or basic public health service accessibility. Besides, the state should also focus on mobile population in the urban. Many policies and financial budgets related to the supply of basic public services are made based on the registered resident population in the region. Therefore, we need to study the basic public services accessibility for mobile population to promote the basic public services development.

\subsection{Enhance the investment of health services, strengthen construction of medical security system}

The state should improve health service quality of remote rural areas and enhance human, finance and material input into basic public health services of remote rural areas, which make them enjoy high quality health service like urban people, thus realizing the purpose of improving the health service accessibility in these regions.

Besides, we should also strengthen the construction of national medical security system, including two benefits: one is enlarging the covering of medical health service to make all the people have the right of enjoying health, thus promoting the spreading of basic health service fairness; another is improving the phenomenon of no cure for disease and relieving the medical burden as well as promoting the health service accessibility.

\subsection{Improve the construction of urban and rural medical assistance system and rural doctor training and welfare compensation mechanism}

Studies have shown that community health service has obvious advantages on accessibility and strengthening the construction of community health service can bring long term beneficial effects. We should reasonably utilize resource on both sides to promote complementary advantages between community health service and comprehensive hospitals, thus providing high quality and convenient health service for residents.[30]
For relatively poor people, they are often unable to gain deserved health service due to poverty, the construction of urban and rural medical assistance system should be improved to relief their medical burden and obtain deserved health service, thus improving their health service accessibility. However, basic medical health service in the rural areas mainly depends on rural doctors with lower level of knowledge and poor welfare. Therefore, the state should establish the access system of rural doctors in rural areas, strengthen training to improve their knowledge level, and improve the corresponding welfare system for the purpose of improving their enthusiasm.

Based on status, promotion of health service accessibility in China has been greatly improved and gained good results. But at present there are many deficits, with the global and Chinese government's increasing attention to this aspect, thus China's health service accessibility will gain a better development in the future.

\section{REFERENCES}

[1] Wang Wei, Ren Ran. Conception and Research Progress of Health Service Accessibility [J]. Chinese Health Economics, 2011, 30(3):47-49.

[2] Kehrer B H, Andersen R, Glaser W A. A Behavioral Model of Families' Use of Health Services[J]. The Journal of Human Resources, 1972, 7(1):125.

[3] Shengelia B, Tandon A, Adams O B, et al. Access, utilization, quality, and effective coverage: An integrated conceptual framework and measurement strategy $[J]$. Social Science \& Medicine, 2005, 61(1):97-109.

[4] Wu Changling, Fang Pengqian. Analysis and Countermeasures of Inequality and Potential Accessibility of Rural Residents' Health Service in Western China [J]. Chinese Health Service Management, 2007, 23(8):560-562.

[5] Dai Kai, Guo Xiaocong. Basic Public Service Accessibility: Conception Definition, Research Progress and Vision.

[6] Huang Jizheng, Li Ke, Chen Meilan. Theory and Practice of Health Service Accessibility [J]. Public Health and Preventive Medicine, 2012, 23(005):6062.

[7] Dai Jiaxin. Research on Accessibility of Western Medical and health Service-Analysis Framework and Comparison $[\mathrm{J}]$. Journal of Tianjin Administration Institute, 2020, v.22; No.206(06):86-96.

[8] Wang Yufeng. Health Service Accessibility Research of Labo Township, Ninglang County, Lijiang City [D]. Yunnan University, 2016. 
[9] Jessop B M, Harvie C J. The influence of stigmatization on access to health services by persons with mental illness, an implication of professional counseling[J]. North American Journal of Fisheries Management, 2013.

[10] Derose K P, Gresenz C R, Ringel J S. Understanding disparities in health care access--and reducing them-through a focus on public health.[J]. Health Affairs, 2011, 30(10):1844-51.

[11] Di M I, Thiede M, Birch S. Access as a policyrelevant concept in low- and middle-income countries.[J]. Health Economics Policy and Law, 2009, 4(Pt 2):179-193.

[12] Charters T J, Harper S, Strumpf E C, et al. The effect of metropolitan-area mortgage delinquency on health behaviors, access to health services, and selfrated health in the United States, 2003-2010[J]. Social Science \& Medicine, 2016, 161:74-82.

[13] Banke-Thomas O E, Banke-Thomas A O, Ameh C A . Factors influencing utilisation of maternal health services by adolescent mothers in Low-and middleincome countries: a systematic review[J]. BMC Pregnancy and Childbirth, 2017, 17(1).

[14] Dimitri, Renmans, Nathalie, et al. Opening the 'black box' of performance-based financing in low- and lower middle-income countries: a review of the literature.[J]. Health policy and planning, 2016, 31(9):1297-309.

[15] Rita, Hamilton, Simon, et al. Utilization and access to healthcare services among community-dwelling people living with spinal cord injury.[J]. Journal of Spinal Cord Medicine, 2017.

[16] Gao Xiang. Research on the Difference of Basic Medical Services Accessibility for the Elderly between Urban and Rural Areas [D]. Huazhong University of Science and Technology, 2017.

[17] Huang Dujuan. Research on the Three-dimensional promotion Model of Basic Public Health Services Accessibility in China [D]. University of Electronic Science and Technology of China, 2018.

[18] Du Benfeng, Han Xiao, Fu Linlin, etc., Demand, Supply, Utilization and Health Promotion Strategy Selection of Medical and Health Services for Mobile Population-Based on the Perspective of Medical Service Utilization Behavior Model [J]. Chinese Journal of Health Policy, 2018(2):23-29.

[19] Jiang Yingying. Research on the Accessibility and Influence Factors of Basic Medical and Health Services for Mobile Population in Shanghai [D]. East China University of Science and Technology, 2019.
[20] Klausner J, Russell M G. Defining Quality of Care[M]. 2020.

[21] Xiao Yizhang, Yang Yuetao, Zhang Ying Feng, etc., Research Progress of Health Service Accessibility [J]. Occupation and Health, 2015, 31(011):15771579 .

[22] Dai Jiaxin. Research on Conception, Measurement and Influence Factors of Accessibility: Literature Review [J]. Study and Practice, 2017(04):86-94.

[23] Meng Kai, Zhang Ling. Five Revisions of Japanese Medical Law and Hospital Management [J]. Chinese Journal of Hospital Administration, 2010, 26(005):397-400.

[24] Dai Mingfeng. Research on Equalization of Basic Medical and Health Services in China [J]. Chinese Journal of Health Informatics and Management, 2018, v.15 (03):106-110.

[25] Xin Yi, He Ning, Zhang Lili. Rural Health Service Accessibility Changes and Obstacles in China [J]. Journal of Changsha Social Work College, 2013, 000(003):23-25.

[26] Hou Jin, Cai Liqiang. Measures and Results of Rural Medical and Health Service Accessibility Reform Based on Problem Orientation [J]. Chinese Primary Health Care, 2019(10).

[27] Xu Ling, Meng Qun. The Second Result of the Fifth National Health Service Survey- Health Service Need, Demand and Utilization; Chinese Journal of Health Informatics and Management.

[28] Hou Jin, Cai Liqiang. Measures and Results of Rural Medical and Health Service Accessibility Reform Based on Problem Orientation [J]. Chinese Primary Health Care, 2019 (10)

[29] Mahapatro S R, James K S , Mishra U S . Intersection of Class, Caste, Gender and Unmet healthcare needs in India: Implications for Health Policy[J]. 2021.

[30] Otis C N. Residency training in anatomic pathology: looking forward in the 21st century.[J]. Human Pathology, 2006, 37(8):929-931. 